Cultures \& Conflits

23 | automne 1996

Circuler, enfermer, éloigner : zones d'attente et centres de rétention aux frontières des démocraties occidentales

\title{
La rétention des étrangers aux frontières françaises
}

\author{
François Julien-Laferrière
}

\section{(2) OpenEdition \\ Journals}

\author{
Édition électronique \\ URL : http://journals.openedition.org/conflits/346 \\ DOI : $10.4000 /$ conflits.346 \\ ISSN : $1777-5345$ \\ Éditeur : \\ CCLS - Centre d'études sur les conflits lilberté et sécurité, L'Harmattan \\ Édition imprimée \\ Date de publication : 15 octobre 1996 \\ ISSN : 1157-996X \\ Référence électronique \\ François Julien-Laferrière, "La rétention des étrangers aux frontières françaises », Cultures \& Conflits \\ [En ligne], 23 | automne 1996, mis en ligne le 13 mars 2006, consulté le 30 mars 2021. URL : http:// \\ journals.openedition.org/conflits/346 ; DOI : https://doi.org/10.4000/conflits.346
}

Ce document a été généré automatiquement le 30 mars 2021.

Creative Commons License 


\title{
La rétention des étrangers aux frontières françaises
}

\author{
François Julien-Laferrière
}

- la première, prévue à l'article 35 bis de l'ordonnance du 2 novembre 1945 relative aux conditions d'entrée et de séjour des étrangers en France, vise les étrangers qui, faisant l'objet d'une mesure d'éloignement - remise à un Etat de la Communauté euro-péenne, arrêté d'expulsion ou arrêté de reconduite -, ne peuvent quitter immédiatement le terri-toire fran-çais ;

5

- la seconde, réglementée par l'article 35 quater de la même ordonnance, concerne les étrangers arrivés par voie ferroviaire, maritime ou aérienne, à qui l'entrée en France est refusée ou dont la demande d'admission sur le territoire au titre de l'asile est en cours d'examen.

6 Ces deux dispositions ont été adoptées à la suite de la révélation de pratiques administratives qui avaient, en leur temps, soulevé quelque émoi. 
- En 1978-1979, est découvert à Arenc, sur le port de Marseille, un hangar dans lequel sont entassés, depuis des semaines ou même des mois, des dizaines d'étran-gers en situa-tion irrégulière, sans aucune com-munication avec l'extérieur ni les moindres conditions de salubrité et d'hygiène, que les autorités de police envisagent de refouler mais qui, pour des raisons diverses - leur identité n'est pas établie avec certitude, aucun bateau n'est dispo-nible vers leur pays de renvoi - , ne peuvent quitter immédiatement la France. Ils sont donc retenus dans ce local précaire, sans garantie juridique aucune - leur statut n'est pas défini, l'autorité ju-diciaire n'est pas informée, la famille n'est pas avertie, ils ne sont pas assisté d'un avocat, etc. -, jusqu'à leur départ qui peut être différé sans limite de temps.

Devant les vives protestations que cette situa-tion de non-droit - au sens propre du terme: aucune règle de droit ne leur est applicablet-, le ministre de l'Intérieur, Christian Bonnet, va introduire dans le projet de loi destiné à modifier l'ordonnance du 2 novembre 1945, une disposi-tion, légalisant cette pratique, qui va instituer la "rétention administra-tive " ${ }^{1}$. Modifiée à plusieurs reprises, elle constitue, depuis la loi du 29 octobre 1981, l'article 35 bis de l'ordonnance.

- Quant à l'article 35 quater, il a une histoire encore plus complexe et intéressante. On trouve là aussi, à l'origine, une pratique qui ne se fonde sur aucun texte, les autorités de police ayant pris coutume de retenir dans la «zone internationale» des aéroports les étrangers auxquels l'entrée du territoire est refusée parce qu'ils ne sont pas munis des documents exigés pour pénétrer en France $^{2}$ ou parce qu'ils demandent l'asile et que leur demande est examinée, afin de déterminer si elle n'était pas destinée à contourner la fer-meture des frontières à l'immigration. Là encore, cette rétention ne connaît aucune limite de temps - des étrangers ont été retenus pendant plusieurs mois - et n'est assortie d'aucune garan-tie. C'est ce qui conduit certains étrangers retenus à l'aéroport de Roissy-Charles de Gaulle à engager à l'encontre du ministre de l'Intérieur, Philippe Marchand, une procé-dure de référé-voie de fait devant le tribunal de grande instance de Paris.

10 Le ministre profite alors de ce qu'un projet de loi relatif à la police des étrangers est en discussion devant le Parlement ${ }^{3}$, pour y greffer une disposition - commu-né-ment appelée " amendement Marchand », du nom de son auteur - permet-tant le maintien en « zone de transit » - nouvelle appellation de la zone internationale - des étrangers non-admis sur le territoire ou des demandeurs d'asile pendant la durée d'examen de leur demande. Mais l'insuffisance des garanties conduit, une fois de plus, le Conseil constitu-tionnel - saisi, ce qui est tout à fait exceptionnel, par le Premier ministre, Edith Cresson - à déclarer cette disposition non-conforme à la Constitution ${ }^{4}$.

11 Le nouveau ministre de l'Intérieur, Paul Quilès, réexamine la question et c'est ainsi que naissent les « zones d'attente » - remplaçant les « zones de transit » mort-nées - des ports et aéro-ports, dont le régime juridique est défini par le nouvel article 35 quater de l'ordonnance du 2 no-vembre 1945, créé à cette occasion ${ }^{5}$. Une loi du 27 décembre 1994 complète cette dis-position, d'une part, pour instituer des zones d'attente dans les "gares ferro-viaires ou-vertes au tra-fic international » et, d'autre part, pour prévoir les conditions du transfert d'un étranger d'une zone d'attente à une autre ${ }^{6}$.

12 Il existe donc deux textes législatifs - les articles 35 bis et 35 quater de l'ordonnance du 2 novembre 1945 - réglementant les hypothèses et les conditions de la rétention ou du 
maintien des étrangers aux frontières françaises. Encore peut-on contester, s'agis-sant de l'article 35 bis relatif à la rétention administrative des étrangers en instance d'éloigne-ment du territoire français, que cette rétention a lieu "aux frontières ", puisque les lieux de rétention sont situés à l'intérieur du territoire français. Mais, dès lors que la mesure est prise pour assurer le départ de l'étranger de France vers un pays de renvoi, la notion de frontière n'est pas loin.

On aurait pu penser que la rétention administrative et le maintien en zone d'attente seraient soumis au même régime juridique. En effet, dans un cas comme dans l'autre, il s'agit d'une mesure privative de liberté - ou, du moins, restrictive de liberté - prise dans le domaine de la police des étrangers et dont le but ultime est le respect de la législa-tion sur l'entrée et le séjour des étrangers en France : la zone d'attente - article 35 quater - est destinée à empêcher l'entrée en France des étrangers qui ne remplissent pas les conditions pour y venir ; la rétention administrative - article 35 bis -, à permettre le départ de ceux qui sont entrés ou séjournent irrégulièrement en France et font l'objet d'un arrêté de recon-duite à la frontière ${ }^{7}$, ou de ceux qui y ont troublé ou risquent d'y troubler l'ordre public et en sont expulsés8.

Les articles 35 bis et 35 quater instituent pourtant deux régimes juridiques bien différents, et ceci pas uniquement en raison des circonstances qui ont présidé à leur rédaction, mais aussi parce qu'il a été souhaité que les deux formes de rétention se distinguent quant au degré de contrainte qu'elles représentent pour les étrangers qui y sont soumis. Cet argument ne manque pas d'être quelque peu spécieux, comme on va tenter de le montrer.

ci de logique - car il faut bien, en général, être entré en France pour pouvoir en être éloigné -, on étudiera successivement le régime du maintien en zone d'attente puis celui de la rétention administrative.

Le maintien en zone d'attente

Les étrangers sont « maintenus » en zone d'attente, ils n'y sont pas « retenus ». C'est ce qu'exprime l'article 35 quater de l'ordonnance du 2 novembre 1945 dont le paragraphe I, alinéa 1er, dispose : «L'étranger qui arrive en France par la voie ferroviaire, maritime ou aérienne et qui soit n'est pas autorisé à entrer sur le territoire français, soit demande son admission au titre de l'asile, peut être maintenu dans une zone d'attente située dans une gare ferroviaire ouverte au trafic international et désignée par arrêté, un port ou un aé-ro-port pendant le temps strictement nécessaire à son départ et, s'il est demandeur d'asile, à un examen tendant à déterminer si sa demande n'est pas manifestement infondée ».

Qu'est-ce que la zone d'attente?

19 Aux termes de l'article 35 quater, paragraphe I, alinéa 3, « la zone d'attente est délimitée par le représentant de l'Etat dans le département [et] s'étend des points d'embarque-ment et de débarquement à ceux où sont effectués les contrôles des personnes ».

20 Cette disposition emporte deux conséquences. D'une part, c'est le représentant de l'Etat - c'est-à-dire le préfet - qui crée une zone d'attente, quand il l'estime nécessaire, dans un port ou un aéroport de son département. En revanche, pour les gares ferro-viaires, l'article 35 quater, paragraphe I, alinéa 1er prévoit que celles qui sont «ouvertes au trafic international » et peuvent, à ce titre, être dotées d'une zone d'attente, sont 
« dési-gnées par arrêté », ce qui semble devoir s'interpréter comme exigeant un arrêté ministériel 9 .

D'autre part, le préfet fixe également les limites de la zone d'attente, dans le cadre que lui impose l'article 35 quater : la zone d'attente comprend l'ensemble des installa-tions fer-roviaires, portuaires et aéroportuaires, depuis les quais ou les aires de stationnement des appareils jus-qu'aux locaux de la police ou des douanes dans lesquels sont contrôlées les personnes et les objets qu'elles transportent avec elles.

Mais l'article 35 quater, paragraphe I, alinéa 3, ajoute que la zone d'attente "peut inclure, sur l'emprise ou à proximité de la gare, du port ou de l'aéroport, un ou plusieurs lieux d'hébergement assu-rant aux étrangers concernés des prestations de type hôtelier ». Il s'agit, dans quelques zones d'attente - essentiellement à Roissy et Orly -, d'hôtels proches de l'aéroport ${ }^{10}$, dont un ou plusieurs étages sont loués par le ministère de l'Intérieur et affectés à l'héber-gement des étrangers maintenus. Dans les autres zones d'at-tente, les étrangers sont maintenus dans des locaux de police situés à l'intérieur du port ou de l'aéroport.

Il existait, fin 1995 un peu plus de cent zones d'attente. Leur nombre exact est diffi-cile à connaître car les préfectures, interrogées par l'Association nationale d'assistance aux frontières pour les étrangers - ANAFÉ ${ }^{11}-$, n'ont pas toutes répondu. Parmi ces zones d'attente, près de $55 \%$ sont des zones portuaires et $45 \%$ seulement des zones aéroportuaires, les zones d'attente ferroviaires, prévues par la loi du 27 décembre 1994 et l'arrêté du 4 mai 1995, n'ayant pas encore été créées ${ }^{12}$.

Par ailleurs, les zones d'attente portuaires semblent actuellement inutiles. En effet, il est de pratique courante, pour ne pas dire systématique, de main-tenir à bord des navires les étrangers arrivant sans les documents exigés pour entrer en France - ceux qu'on appelle les «passagers clandestins » -, qu'ils demandent l'asile ou non. Ils sont en général renvoyés sur le navire à bord duquel ils sont arrivés et, en cas de demande d'asile, celle-ci est examinée sans que l'intéressé soit autorisé à débarquer. Cette pratique, qui ne repose sur aucun texte et constitue, au contraire, une méconnaissance de l'article 35 quater de l'ordonnance du 2 novembre 1945, a été qualifiée de voie de fait à plusieurs reprises par les tribunaux judiciaires ${ }^{13}$, sans toutefois que ces condamnations réitérées conduisent les autorités de police aux frontières, agissant sur instructions du ministère de l'Intérieur, à mettre fin à ces agissements ${ }^{14}$.

Qui peut être maintenu en zone d'attente?

article 35 quater, paragraphe I, alinéa 1er, mentionne deux catégories d'étrangers qui peuvent être maintenus en zone d'attente: les «non-admis» - c'est-à-dire les étrangers auxquels l'entrée en France est refusée - et les demandeurs d'asile. Les premiers sont maintenus "pendant le temps strictement nécessaire à [leur] départ »; les seconds, pendant la durée d'un examen "tendant à déterminer si [leur] demande [d'asile] n'est pas manifestement infondée ».

Les « non-admis »

Il s'agit des étrangers qui viennent en France pour tout autre motif que l'asile - tourisme, visite familiale, séjour professionnel, études, etc. - et à qui l'entrée du territoire est refusée, ou bien parce qu'ils n'ont pas les documents exigés ${ }^{15}$, ou bien parce que leur "présence constituerait une menace pour l'ordre public», ou enfin parce qu'ils font « l'ob-jet soit d'une interdiction du territoire, soit d'un arrêté d'expulsion » ${ }^{16}$. 
- elle ne peut être prise que par le chef du poste frontière ou un fonctionnaire désigné par lui et ayant au moins le grade d'inspecteur, s'il s'agit d'un fonctionnaire de police, ou de contrôleur, s'il s'agit d'un fonctionnaire des douanes ${ }^{17}$;

31

- elle doit prendre la forme "d'une décision écrite [...], spécialement motivée d'après les éléments de l'espèce, dont le double est remis à l'intéressé " ${ }^{18}:$ la décision doit donc énoncer «les considérations de fait et de droit qui [en] constituent le fondement » ${ }^{19}$, notamment celle des conditions légales exigées pour l'entrée du territoire à laquelle l'inté-ressé ne satisfait pas ;

32

- elle ne peut être exécutée «contre le gré de l'intéressé avant l'expiration du délai d'un jour franc $»{ }^{20}$, délai pendant lequel l'étranger « est mis en mesure d'avertir ou de faire avertir la personne chez laquelle il a indiqué qu'il devait se rendre, son consulat ou le conseil de son choix ${ }^{21}$.

d'origine ou vers un pays tiers l'étranger à qui l'entrée est refusée - qui a conduit à pré-voir le maintien en zone d'attente « pendant le temps strictement nécessaire à son départ ». roviai-re ouverte au trafic international, sans être muni des documents et justificatifs exigés ${ }^{22}$, et qui demandent à être néanmoins admis à entrer sur le territoire " au titre de l'asile ». En effet, même en l'absence de toute disposition législative expresse en ce sens, les deman-deurs d'asile sont dispensés de l'obligation de passeport et de visa et l'entrée du terri-toire ne peut donc leur être refusée au seul motif qu'ils ne sont pas munis des docu-ments exigés ${ }^{23}$.

Cette dispense est fondée sur l'article 31 de la Convention de Genève du 21 juillet 1951 relative au statut des réfugiés, qui interdit aux Etats contractants d'appliquer "des sanctions pénales, du fait de leur entrée ou de leur séjour irréguliers aux réfugiés qui, arrivant direc-tement du territoire où leur vie ou leur liberté était menacée (...), entrent ou se trouvent sur leur territoire sans autorisation ", le bénéfice de cette stipulation étant étendue aux deman-deurs d'asile jusqu'à ce qu'une décision définitive soit prise sur leur qualité de réfugié.

Etats examinent à la frontière la demande d'asile qui leur est présentée et refusent l'entrée du territoire quand la demande apparait "manifestement infondée " ${ }^{24}$. Le maintien en zone d'attente est donc destiné à permettre l'examen de la demande d'asile - sans empor-ter entrée sur le territoire - et le départ de l'intéressé si, à l'issue de cet examen, sa demande est considérée comme « manifestement infondée ». 
- Qu'est-ce qu'une «demande d'asile manifeste-ment infondée »? Cette notion peut s'entendre - et, dans la pratique, s'entend - dans des sens divers : d'une part, une demande est manifestement infondée quand l'intéressé ne fait état d'aucun risque encouru dans son pays d'origine et qu'il en ressort qu'il n'a pas besoin de protection : la demande d'asile manque alors effectivement de fondement; d'autre part, une demande est égale-ment déclarée manifestement infondée - bien qu'alors elle ne soit pas examinée au fond - quand l'intéressé peut obtenir la protection d'un autre Etat que la France, notam-ment si, entre son pays d'origine et son arrivée en France, il est passé par un Etat auquel il aurait pu demander l'asile et qui était susceptible de le lui accorder (théorie du pays tiers d'accueil).

- Qui apprécie si la demande d'asile est «manifestement infondée »? C'est la Division asile aux frontières (DAF), dépendant du ministère des Affaires étrangères et composée d'agents de l'OFPRA détachés à cet effet. Les agents de la DAF entendent les demandeurs d'asile maintenus en zone d'attente et formulent un avis sur le caractère manifeste-ment infondé ou non de leur demande, cet avis étant transmis au ministre des Affaires étrangères.

41 Si la demande d'asile est considérée comme mani-festement infondée, une décision de refus d'entrée est prise par le ministre de l'Intérieur prise après consul-tation du ministre des Affaires étrangères . ${ }^{25}$. L'intéressé est alors renvoyé, soit vers son pays d'origine si l'examen de sa demande d'asile a permis de conclure qu'il n'y court pas de risque, soit vers un « pays tiers d'accueil » s'il apparaît qu'il aurait pu y solliciter l'asile avant son arrivée en France et qu'il y bénéficiera d'une protection, soit enfin vers l'Etat partie à la Convention de Schengen qui, en application des stipulations de celles-ci, est responsable de l'examen de sa demande d'asile.

$\mathrm{Si}$, au contraire, la demande d'asile n'est pas jugée comme manifestement infondée, il est délivré à l'intéressé un sauf-conduit, dont la validité varie de quarante-huit heures à une semaine, sous couvert duquel il peut pénétrer sur le territoire français pour se rendre à la préfecture dans le ressort de laquelle il fixera sa résidence, en vue d'y présenter une demande d'admission au séjour, en application de l'article 31 bis de l'ordonnance du 2 novembre 1945, puis une demande de reconnais-sance de la qualité de ré-fugié auprès de l'OFPRA dans les conditions définies par l'article 32 de l'ordonnance.

Les étrangers en « transit interrompu »

Il faut ajouter, à ces deux catégories, celle - reléguée pour des raisons obscures au paragraphe VII du même article - des « étranger[s] qui se trouve[nt] en transit dans une gare, un port ou un aéroport si l'entreprise de transport qui devait [les] acheminer dans le pays de destination ultérieure refuse de [les] embarquer ou si les autorités du pays de destination [leur] ont refusé l'entrée et [les] ont renvoyé[s] en France ». C'est ce qu'on appelle le «transit interrompu », qui vise principalement deux hypothèses :

- l'étranger qui n'est pas muni des documents exigés pour l'entrée dans le pays de sa destination finale et que, pour éviter des difficultés à l'arrivée dans ce pays, le commandant de bord refuse d'embarquer ; 
- celui qui s'est déjà présenté à l'entrée du pays de sa destination finale mais que les autorités de ce dernier ont renvoyé en France, d'où il provenait, parce qu'il n'était pas muni des documents exigés pour entrer.

La situation de ces étrangers n'est pas définie dans la loi en tant que telle. Elle n'est qu'évoquée dans le cadre de l'article 35 quater, paragraphe VII, qui prévoit qu'ils peuvent être maintenus en zone d'attente. Une circulaire du ministre de l'Intérieur du 9 juillet 1992, prise pour l'appli-cation de la loi du 6 juillet 1992 ${ }^{26}$, précise toutefois $q^{\prime}$ '« aucu-ne décision de refus d'admis-sion n'est prononcée, sauf s'ils demandent à péné-trer sur le territoire français sans rem-plir les conditions d'entrée ou s'ils ont refusé d'embar-quer », et que «la procédure rela-tive aux demandeurs d'asile ne leur est pas applicable, sauf s'ils demandent l'asile ».

Ce qui signifie, en clair, qu'ils ne bénéficient d'aucune garantie et peuvent être renvoyés sans délai ${ }^{27}$, sans formalité, sans qu'il en reste aucune trace. Aucune décision ne leur est notifiée, puisqu'il n'en est pris aucune. Il ne peuvent donc prévenir personne puisqu'ils ne savent pas à l'avance quel sort leur sera réservé. Seule l'absence de vol dans un bref délai peut conduire à décider leur maintien en zone d'attente, cette décision mentionnant simplement "les circons-tances qui ont conduit à porter la situation de l'inté-ressé à la connaissance du service de contrôle » et « les raisons qui empêchent un départ immédiat ${ }^{28}$.

Selon les statistiques du ministère de l'Intérieur ${ }^{29}$, les étrangers arrivant en France par voie maritime ou aérienne - quelque 35.000 - ne représentent qu'à peine $4 \%$ du total des entrées en France et les personnes maintenues en zone d'attente ne seraient qu'environ 2.500 chaque année, dont un peu plus de 450 demandeurs d'asile. Parmi ces derniers, près de la moitié (46 \% exactement en 1994) auraient été admis à entrer sur le territoire. De plus, la durée d'instruction des dossiers de demandes d'asile, depuis l'enregistrement de la demande jusqu'à la notification de la décision, est de 3,61 jours, ce qui implique que le maintien de la majorité des demandeurs d'asile n'est pas prolongé et que leur cas n'est donc pas examiné par le juge judiciaire ${ }^{30}$. Le régime du maintien en zone d'attente

51 Il est longuement décrit à l'article 35 quater, paragraphes II à $\mathrm{V}$, de l'ordonnance du 2 novembre 1945 qui pose des règles de compétence, de délai et de recours, et qui définit les droits des étran-gers pendant leur maintien en zone d'attente.

Le maintien en zone d'attente et sa prolongation: compétence administrative et compétence judiciaire

La décision de maintien en zone d'attente est prise par le « chef du service de contrôle aux frontières ou un fonctionnaire désigné par lui, titulaire au moins du grade d'inspec-teur ", règle de compétence identique à celle relative au refus d'entrée sur le territoire. La décision de maintien doit être écrite et motivée. Elle est inscrite sur un registre auquel le procureur de la République a accès quand il le demande.

La décision de maintien et sa prolongation:

Le maintien est prononcé pour une durée maximum de quarante-huit jours, renouvelable une fois selon les mêmes modalités. Au-delà de quatre jours, le maintien ne peut être prolongé, pour une durée maximum de huit jours, que par ordonnance du président du tribunal de grande instance (TGI) sur le territoire duquel la zone d'attente est située, ou d'un magistrat du siège délégué par lui. Une nouvelle prolongation de 
huit jours au plus peut être autorisée, «à titre exceptionnel», dans les mêmes conditions.

Le président du TGI est saisie par le chef du service de contrôle aux frontières, avant l'expiration du délai de quatre jours, ou de douze jours s'il s'agit d'une seconde prolongation. L'administration « expose dans sa saisine les raisons pour lesquelles l'étranger n'a pu être rapatrié ou, s'il a demandé l'asile, admis, et le délai nécessaire pour assurer son départ de la zone d'attente».

Le rôle du juge judiciaire :

tion se pose de savoir quels pouvoirs cette disposition confère au juge judiciaire.

Il convient, à cet égard, de distinguer selon que l'étranger est un non-admis ou un demandeur d'asile :

59

- Dans le premier cas, le principe de séparation des autorités administratives et judiciaires s'oppose à ce que le président du TGI contrôle la déci-sion de refus d'entrée en France, celle-ci étant prise par une autorité administrative et relevant donc de la seule juridiction administrative ${ }^{31}$. Le juge judiciaire ne peut alors, « connaissance prise des rai-sons du refus [...] opposé à l'intéressé par l'administration, et du délai nécessaire pour assurer son départ, que statuer sur [son] maintien au-delà de quatre jours $»^{32}$.

60 En d'autres termes, le juge ne peut remettre en cause l'appréciation portée par l'autorité administrative sur la situation de l'étranger au regard des conditions d'entrée en France; il peut seulement vérifier si un délai est nécessaire pour son départ de la zone d'attente et autoriser la prolongation du maintien pendant le temps nécessaire à ce départ, c'est-à-dire la plupart du temps jusqu'au prochain vol.

61

- Quand l'étranger maintenu est un demandeur d'asile et que l'administration n'a pas encore statué sur le caractère "manifestement infondé » ou non de sa demande, le rôle $\mathrm{du}$ président du TGI semble devoir être tout autre. En effet, dans ce cas, l'administration n'a pas encore pris de décision, le principe de séparation des autorités n'est donc pas en cause.

Mais en outre, l'article 35 quater n'autorisant le maintien du demandeur d'asile en zone d'attente que "pendant le temps strictement nécessaire [...] à un examen tendant à déterminer si sa demande n'est pas manifestement infondée ", le juge judiciaire institué gardien de la liberté individuelle par l'article 66 de la Constitution et intervenant à ce titre en cette matière - ne devrait prolonger le maintien que lorsqu'il n'est pas en mesure d'apprécier lui-même si la demande d'asile n'est pas manifestement infondée. S'il est, en revanche, en mesure de le faire et s'il estime que la demande n'est pas manifestement infondée, il doit refuser de prolonger le maintien, sauf à porter lui-même une atteinte injustifiée à la liberté individuelle et, partant, à violer l'article 66 de la Constitution ${ }^{33}$.

Les pouvoirs du juge sont donc "à géométrie variable »: simple rôle de constatation des possibilités de départ pour les non-admis - on l'a comparé, dans ce cas, à un « agent de voyage » qui se borne à consulter les horaires des compagnies aériennes et à proposer à l'administration de faire partir le non-admis par le premier vol disponible... -, il est, à l'inverse, un véritable organe de décision en matière d'asile puisque c'est lui 
qui, en fin de compte, autorise l'entrée du territoire s'il estime que la demande n'est pas manifestement infondée.

- Dès qu'est prise la décision de maintien, l'étranger « est immédiatement informé de ses droits et de ses devoirs, s'il y a lieu par l'intermédiaire d'un interprète ", « mention en est faite sur le registre [...] qui est émargé par l'intéressé » et qui consigne « l'état civil de l'intéressé et la date et l'heure auxquelles la décision de maintien lui a été notifiée »; cette décision « est portée sans délai à la connaissance du procureur de la République ».

73 L'assistance d'un interprète est donc de droit et c'est l'administration qui doit faire le nécessaire pour l'assurer à l'étranger, sans que celui-ci doive la solliciter ni, a fortiori, en supporter les frais. Il est parfois difficile de recourir à un interprète dans un très bref délai, si bien qu'il n'est pas rare que l'interprète assiste l'étranger "à distance ", par télé-phone, ce qui n'est pas sans présenter de sérieux inconvénients.

Les voies de recours :

faire l'objet d'un recours en annulation devant le tribunal administratif du ressort. Ce recours est, toutefois, dénué de toute efficacité puisqu'il n'a pas d'effet suspensif - le requérant est donc maintenu malgré son recours - et qu'en outre il ne sera jamais examiné avant l'expiration du délai du maintien - au maximum vingt jours.

après quatre jours comme après douze jours -, elles peuvent être déférées, par l'intéressé, le ministère public ou le préfet, «dans les quatre jours » de leur prononcé ${ }^{34}$, au «premier président de la Cour d'appel ou son délégué [qui] doit statuer dans les quarante-huit heures de sa saisine ${ }^{35}$. L'ordonnance d'appel peut faire l'objet d'un pourvoi en cassation, par l'étranger, le préfet ou le ministère public, « dans les dix jours suivant (sa) notification ${ }^{36}$.

l'utilité. Cependant, vu la brièveté des délais d'appel - le juge d'appel peut statuer avant l'expiration du délai de maintien -, cette voie est fréquemment employée. Pour éviter que l'ordonnance de première instance autorisant la prolongation du maintien ne soit infirmée - ce qui oblige à faire entrer l'étranger sur le territoire -, l'administration ne recule pas à précipiter le départ de l'intéressé vers son pays d'origine - ou, parfois même, vers un pays tiers -, en empruntant, si besoin, des itinéraires surprenant, que seule explique la disponibilité d'un vol. Le juge d'appel ne peut alors que constater que l'étranger n'est plus en zone d'attente et qu'il n'y a donc pas lieu de statuer sur son maintien...

Les droits des étrangers maintenus matérielles, d'autre part.

Les garanties juridiques :

Elles sont énumérées de façon détaillée par l'article 35 quater, paragraphes I et V, de l'ordonnance du 2 novembre 1945 :

Le registre, que le procureur de la République peut consulter à tout moment, permet de vérifier que les délais - quarante-huit heures pour le renouvellement de la décision ad- 
ministrative ; saisine du président du TGI au bout de quatre jours - sont effectivement respectés.

- Pendant la durée du maintien, l'étranger maintenu « peut demander l'assistance d'un interprète et d'un médecin et communiquer avec un conseil ou toute personne de son choix ». gné par les fonctionnaires de police chargés de la surveillance de la zone d'attente ; il s'agit, par exemple, du médecin de garde de l'aéroport ou du port. En pratique, il semble que les demandes de visite d'un médecin sont fréquemment rejetées et, quand l'étranger est mis en mesure d'exercer ce droit, il est emmené au service médical ou, s'il est dans l'incapacité de s'y rendre, le médecin se déplace jusqu'au lieu du maintien. Le médecin peut examiner l'étranger, lui donner des médicaments de base et établir des certificats médicaux ${ }^{37}$.

77 Le choix d'un conseil pose une double difficulté. D'abord, quant au sens du mot « conseil»: doit-il nécessairement s'agir d'un avocat ou peut-il s'agir de toute autre per-sonne susceptible de venir en aide à l'intéressé, tel un représentant d'association humani-taire, un agent consulaire, etc.? Ensuite, comment l'intéressé peut-il effectivement exer-cer son droit s'il ne connaît aucun avocat ou aucune association humanitaire? Ainsi, la liste des avocats du barreau du Val d'Oise est affichée à l'hôtel Ibis de Roissy, mais est-elle d'une quelconque utilité pour les étrangers maintenus, dès lors que, d'une part, le TGI compétent est celui de Bobigny (Seine-Saint-Denis) et non celui de Pontoise, et que, d'autre part, cette liste n'indique pas quels sont les avocats spécialistes du droit des étran-gers ou disposés à assister les étrangers en zone d'attente.

On peut, par ailleurs, s'interroger sur la portée de la conjonction «ou », qui peut s'interpréter - et est en fait parfois interprétée - comme n'autorisant l'étranger à entrer en contact qu'avec une seule personne : un conseil «ou » toute autre personne, mais non un conseil « et » toute autre personne.

comment l'étranger va-t-il, en pratique, communiquer avec un conseil ou une autre personne ? Une cabine téléphonique est, à cet effet, mise à la disposition des personnes maintenues dans certaines zones d'attente, notamment à Roissy et Orly. Mais cette cabine ne peut être utilisée que par les détenteurs de cartes téléphoniques et les cartes sont vendues aux seuls possesseurs de francs français.

Si le droit de communiquer avec l'extérieur est prévu par la loi, on voit que son exercice relève de la course d'obstacles.

- Le procureur de la République et le président du TGI ou un juge délégué par lui peuvent - le premier pendant toute la durée du maintien et les seconds après les quatre premiers jours - «se rendre sur place pour vérifier les conditions de ce maintien et se faire communiquer le registre ».

Quant on sait la lourdeur des charges du procureur de la République et du président du TGI, on mesure le caractère illusoire de cette garantie : le procureur de la République ne peut faire la tournée des commissariats où s'effectuent les gardes à vue, des centres de rétention où sont placés les étrangers en voie d'éloignement du territoire ${ }^{38}$, et des zones d'attente, son temps n'y suffirait pas. De mémoire d'étranger maintenu en zone

Cultures \& Conflits, 23 | automne 1996 
d'attente, d'ailleurs, on ne se souvient avoir eu la visite d'un procureur de la République...

- D'une part, l'article 35 quater ne mentionne l'existence de «lieux d'hébergement assurant [...] des prestations de type hôte-lier» que comme une possibilité et non comme une obligation : le texte porte en effet " peut in-clure » et non " inclut ». Est-ce à dire que ces prestations ne sont offertes aux étrangers maintenus que lorsque des lieux d'héber-gements spécifiques le permettent ou, au contraire, que ces prestations doivent, en tous les cas, être offertes, le cas échéant dans des lieux distincts de la zone s'étendant « des points d'embarquement et de débarquement à ceux où sont effectués les contrôles des personnes »?

89

- D'autre part, dans quelles conditions ces prestations sont-elles assurées ? Les étrangers maintenus ont-ils droit à la préservation de leur intimité, à séjourner dans des pièces qu'ils peuvent fermer, où ils séjournent seuls ou avec les seuls membres de leur famille, ou doivent-ils accepter que les pièces restent ouvertes, afin de permettre l'exercice d'un contrôle par les personnels chargés d'assurer la surveillance ; doiventils accepter de par-tager leur lieu d'hébergement avec des personnes qui leur sont ... étrangères et n'avoir ainsi aucune possibilité de préserver leur intimité individuelle ou familiale; les membres d'une même famille ont-ils droit de séjourner ensemble ou l'administra-tion a-t-elle le droit de les séparer, de regrouper les hommes d'un côté, et les femmes de l'autre ; quel est le sort des enfants, etc. ${ }^{40}$ ?

L'Office des migrations internationales (OMI) ${ }^{41}$ est présent en zone d'attente, à Roissy et à Orly, par l'intermédiaire d'un ou plusieurs agents dont le rôle est d'apporter une aide humanitaire aux étrangers maintenus. Concrètement, les représentants de l'OMI 
se bornent à vendre des cigarettes, des cartes téléphoniques, de la nourriture. Leur action est donc purement matérielle; ils n'apportent aucune assistance juridique aux étrangers maintenus.

\section{L'accès aux zones d'attente}

L'article 35 quater, paragraphe $\mathrm{V}$, permet à l'étranger maintenu en zone d'attente de " communiquer avec un conseil ou toute personne de son choix », mais il ne précise pas si le conseil et la personne contactés par l'étranger peuvent venir lui rendre visite en zone d'attente. En re-vanche, le même paragraphe prévoit qu'« un décret en Conseil d'Etat détermine les conditions d'accès du délégué du haut-commissariat des Nations unies pour les réfugiés ou de ses représentants ainsi que des associations humanitaires à la zone d'attente ».

Les visites des avocats et des personnes privées :

En pratique, il est difficile aux avocats de rendre visite à leurs clients quand ceux-ci, après les avoir contacté par téléphone ou par l'intermédiaire d'un tiers, souhaitent s'entretenir directement avec eux. En l'absence de toute précision dans l'ordonnance de 1945 ou dans un texte d'application, les conditions d'accès des avocats aux zones d'attente sont variables selon les plus ou moins bonnes dispositions des fonctionnaires de police, qui accordent ou non un droit de visite, celui-ci s'assimilant parfois aux conditions de visite aux détenus dans les prisons.

Pour les autres visites, qui ne sont pas davantage réglementées par les textes, la demande doit en être faite à l'officier de quart qui donne ou non son accord. Selon les cas, le droit de visite peut être une simple formalité ou un véritable «parcours du combat-tant ». En effet, il est soumis au bon vouloir de l'officier de quart qui en limite parfois arbi-traire-ment la durée.

L'accès du HCR et des associations humanitaires :

Le décret prévu à l'article 35 quater, paragraphe $V$, alinéa 2, n'est intervenu que le 2 mai 1995, soit près de trois ans après la promulgation de la loi du 6 juillet $1992^{42}$. Il distingue le régime de l'accès du délégué du haut-commissariat des Nations unies pour les réfugiés (HCR) de celui des associations.

L'accès à la zone d'attente du délégué du HCR ou de ses représentants est subordonné à un agrément individuel délivré pour un an par le ministre de l'Intérieur après avis du ministère des affaires étrangères. Le ministre de l'Intérieur garde le droit de retirer son agrément, ce retrait devant être motivé. Les modalités pratiques de l'accès dans chaque zone d'attente seront arrêtées d'un commun accord entre le délégué du HCR et le ministère de l'Intérieur « de manière à permettre l'exercice effectif de sa mission par le haut-commissariat des Nations unies pour les réfugiés ».

Sur place, le délégué du HCR ou ses représentants peuvent s'entretenir avec le chef du service de contrôle aux frontières et, lorsqu'ils sont présents, avec les représentants du minis-tère des Affaires étrangères (DAF) et de l'OMI, ainsi que "confidentiellement » avec les deman-deurs d'asile maintenus en zone d'attente, mais non avec les étrangers qui n'ont pas demandé l'asile.

Enfin une réunion annuelle est organisée, à l'initiative du ministère de l'Intérieur, avec le délégué du HCR et ses représentants, sur le fonctionnement des zones d'attente.

Les associations habilitées à proposer des représentants en vue d'accéder à la zone d'attente sont désignées par arrêté du ministre de l'Intérieur. L'habilitation ne peut 
être sollicitée que par les associations régulièrement déclarées depuis cinq ans et se proposant par leur statut l'aide et l'assistance aux étrangers, la défense des droits de l'homme ou l'assistance médicale et sociale. L'habilitation est accordée pour deux ans et est renouvelable pour la même durée. Tout refus d'habilitation doit être motivé (article 7).

Un arrêté du ministre de l'Intérieur du 7 décembre $1995^{43}$ a fixé la liste des associa-tions habilitées. Elles sont cinq : la Croix rouge française ; l'Association nationale d'assistance aux frontières pour les étrangers (ANAFÉ) ; Amnesty international - section française ; France terre d'asile ; la Cimade.

L'accès des représentants des associations est subordonné à un agrément individuel délivré pour un an par le ministre de l'Intérieur après avis du ministère des Affaires étrangères. Cet agrément, qui est renouvelable, ne peut être accordé qu'à cinq personnes par association. Le ministre de l'Intérieur peut, également après avis du ministre des Affaires étrangères, retirer l'agrément délivré à une association ou à un représentant d'une association, la décision de retrait devant, dans les deux cas, être motivée.

Une même association ne peut accéder, par l'intermédiaire de deux représentants au plus, à une même zone d'attente qu'une fois par trimestre et entre 8 heures et 20 heures seulement. Le ministre de l'Intérieur peut autoriser toute visite supplémentaire sur demande écrite et motivée du président de l'association ou de tout membre mandaté.

Les représentants des associations peuvent s'entretenir confidentiellement avec les personnes maintenues dans la zone mais, pendant leur présence en zone d'attente, ils sont accompagnés par un agent des services de contrôle aux frontières.

Ce décret appelle diverses observations :

107

- en ce qui concerne l'accès du HCR, il précise que ses représentants pourront s'entretenir uniquement avec les personnes maintenues qui ont demandé leur admission sur le territoire au titre de l'asile; or, la pratique montre qu'un certain nombre d'étrangers éprouvent des difficultés à faire enregistrer leur demande d'asile et même à la formuler ; un contact avec le HCR aurait été utile à ces personnes ;

- une même association ne peut avoir accès à une même zone d'attente que douze heures sur vingt-quatre et qu'une seule fois par trimestre; on est bien loin de la possibilité, pour les associations, d'être présentes en zone d'attente « dans la mesure où [elles voudraient] l'être », ainsi que l'avait souhaité le Sénat lors du vote de la loi du 6 juillet $1992^{44}$;

109

- le décret ne permet pas l'accès le même jour à la même zone d'attente de représentants de différentes associations; ainsi, dans le cas d'une arrivée massive d'étrangers d'un même pays, l'efficacité de l'action des associations sera extrêmement limitée ;

110

- le décret paraît contradictoire puisque les représentants des associations ont la possibilité de s'entretenir "confidentiellement» avec les étrangers maintenus, mais qu'ils sont simultanément «accompagnés par un agent des services de contrôle aux 
frontières "; la confidentialité d'un entretien entre deux personnes - ici le représentant de l'association et l'étranger maintenu - exclut la présence d'un tiers, d'autant plus que cette confiden-tialité sera, semble-t-il respectée pour les représentants du HCR qui, eux, ne sont pas accom-pagnés pendant leur visite en zone d'attente ${ }^{45}$.

La fin du maintien en zone d'attente

Le maintien en zone d'attente peut prendre fin pour des raisons diverses.

113

- D'abord, l'étranger maintenu peut quitter volontairement la zone d'attente "pour toute destination située hors de France ", comme l'y autorise l'article 35 quater, paragraphe II, alinéa 2, de l'or-donnance du 2 novembre 1945. Il s'agira alors, la plupart du temps, d'un départ vers le pays d'origine, car il est extrêmement rare que l'étranger maintenu en zone d'attente soit admissible dans un autre pays. En pratique, cette hypothèse de départ volontaire ne peut qu'être tout à fait exceptionnelle et aucun exemple n'a été recensé.

- Il est donc plus fréquemment mis fin au maintien par l'administration, parce que le départ de l'étranger peut être réalisé. Ce départ s'analyse alors en l'exécution de la décision de refus d'entrée ; elle est donc soumise au régime décrit plus haut ${ }^{46}$.

- Le maintien peut encore cesser en cas d'admission de l'intéressé sur le territoire français, quand la demande d'asile n'est pas jugée "manifestement infondée », ou quand la décision de refus d'entrée dont il était l'objet est rapportée, par exemple si l'authenticité d'un document, contestée dans un premier temps, est finalement établie, ou si l'adminis-tration accepte de délivrer à l'étranger un " visa de régularisation » lui permettant d'entrer sur le territoire en vue de s'y faire délivrer un titre de séjour.

- Enfin, le maintien cesse à l'expiration du dernier délai autorisé, soit que le juge judiciaire a refusé de prolonger le maintien en zone d'attente, soit que, à l'expiration du délai maximum de vingt jours autorisé par l'article 35 quater, paragraphes III et IV, de l'ordonnance du 2 novembre 1945, le départ de l'étranger vers son pays de provenance ou de renvoi n'est pas possible ou qu'il n'a pas été statué sur le caractère manifestement infondé ou non de sa demande d'asile.

Dans cette hypothèse, en application de l'article 35 quater, paragraphe VI, l'administration doit délivrer à l'étranger un sauf-conduit, qui lui permet d'entrer sur le territoire et qui est valable pour une durée de huit jours, au terme de laquelle il devra avoir quitté le territoire ou régularisé sa situation auprès de la préfecture en obtenant une autorisation provisoire de séjour ou un récépissé de demande de carte de séjour. 


\section{NOTES}

1. Loi $n^{\circ} 80-9$ du 10 janv. 1980 (dite loi Bonnet), partiellement déclarée non-conforme à la Constitution par le Conseil constitutionnel (décision n ${ }^{\circ}$ 79-109 DC du 9 janv. 1980). 2. La liste des documents exigés pour entrer en France figure à l'art. 5 de l'ord. du 2 nov. 1945 qui dispose : « Pour entrer en France, tout étranger doit être muni : $1^{\circ}$ des documents et visas exigés par les conventions internationales et les règlements en vigueur $; 2^{\circ}$ sous réserve des conventions interna-tionales, des documents [...] relatifs, d'une part, à l'objet et aux conditions de son séjour et, d'autre part, s'il y a lieu, à ses moyens d'existence et aux garanties de son rapatriement $; 3^{\circ}$ des documents nécessaires à l'exer-cice d'une activité professionnelle s'il se propose d'en exercer une ».

3. Il s'agissait principalement d'introduire un mécanisme de responsabilité des transporteurs acheminant en France des étrangers non munis des documents exigés et d'adapter l'ord. du 2 nov. 1945 aux conditions posées par la Convention de Schengen en matière d'entrée sur le territoire et d'éloignement des étrangers en situation irrégulière. Ce projet de loi allait devenir la loi n 92-190 du 26 févr. 1992.

4. C. const. 25 févr. 1992, décision $n^{\circ}$ 92-307 DC.

5. Loi n $92-625$ du 6 juill. 1992.

6. Loi $n^{\circ}$ 94-1136 du 27 déc. 1994. Compte tenu de la place limitée dont on dispose ici, on n'étudiera pas les modalités du transfert d'une zone d'attente à une autre, qui ont un aspect essentiellement technique et ne posent pas de problèmes principe aussi délicats que le principe même du maintien en zone d'attente.

7. Ord. du 2 nov. 1945, art. 22 : « Le représentant de l'Etat dans le département [i.e. : le préfet] et, à Paris, le préfet de police peuvent, par arrêté motivé, décider qu'un étranger sera reconduit à la frontière dans les cas suivants : $1^{\circ}$ si l'étranger ne peut justifier être entré régulièrement sur le territoire français [...] ; $2^{\circ}$ si l'étranger s'est maintenu sur le territoire au-delà de la durée de validité de son visa ou [...] à l'expiration d'un délai de trois mois à compter de son entrée sur le territoire, sans être titulaire d'un titre de séjour régulièrement délivré ; $3^{\circ}$ si l'étranger, auquel la délivrance ou le renouvellement d'un titre de séjour a été refusé ou dont le titre de séjour a été retiré, s'est maintenu sur le territoire au-delà du délai d'un mois à compter de la date de la notification du refus ou du retrait [...] ».

8. Ord. du 2 nov. 1945, art. 23 : « L'expulsion peut être prononcée par arrêté du ministre de l'Inté-rieur si la présence sur le territoire français d'un étranger constitue une menace grave pour l'ordre public »; art. 26 : «L'expulsion peut [également] être prononcée : a) en cas d'urgence absolue $[. .$.$] ; b) lors-qu'elle constitue une nécessité$ impérieuse pour la sûreté de l'Etat ou la sécurité publique [...] ».

9. C'est d'ailleurs un arrêté du ministre de l'Intérieur du 4 mai 1995 qui désigne « les gares ferroviaires ouvertes au trafic international au sens de l'article 35 quater de l'ordonnance du 2 novembre 1945 ». Ces gares sont (art. 1er) : Lille-Europe, LilleFlandres, Aulnoye, Strasbourg, Thionville, Forbach, Metz, Sarreguemines, Pontarlier, Morteau, Modane, Cerbère, Nice, Hendaye, Paris-Nord, Paris-Est, Paris-Lyon. L'art. 2 de cet arrêté ajoute : «Les préfets et, à Paris, le préfet de police peuvent, en cas de nécessité et pour un délai limité, désigner comme gare ferroviaire ouverte au trafic international au sens de l'article 35 quater de l'ordonnance du 2 novembre 1945 toute 
gare située dans leur département ». Cette disposition a fait l'objet d'un recours actuellement pendant $†-$ devant le Conseil d'Etat.

10. Ces hôtels, anciennement Arcade, appartiennent maintenant à la chaîne Ibis. 11. Cette association - qui regroupe des organisations de défense des droits de l'homme et des étrangers, telles que Amnesty international, la Cimade, France terre d'asile, le GISTI, le MRAP, la Ligue des droits de l'homme, et des organisations syndicales des personnels d'Air France et d'Aéroport de Paris - a pour objet social l'aide aux étrangers éprouvant des difficultés à se faire admettre sur le territoire français, notamment dans les zones d'attente. Elle a adressé, en juillet 1995, à toutes les préfectures de la France métropolitaines et des DOM une demande de communication de la liste des zones d'attente créées dans le département; elle n'a obtenu que 53 réponses. Les chiffres cités ici se fondent, d'une part, sur ces réponses et, pour le reste, sur les informations communiquées par le ministère de l'Intérieur à la Commis-sion nationale consultative des droits de l'homme (CNCDH) le 23 novembre 1993.

12. Le nombre de cent zones d'attente, environ, doit être appréhendé avec prudence. En effet, le préfet de la Guadeloupe a créé dans son département vingt zones d'attente, probablement pour parer à tout risque d'immigration clandestine par voie maritime. Par ailleurs, il existe des zones d'attente dans des aéroports tels que ceux de Lannion, Dole, Cholet ou Brive-la-Gaillarde, qui ne sont sans doute pas promises à une très grande fréquentation.

13. Voir notamment : TGI Paris, 29 juin 1994, UDAF et Zito Mwinyi c. Min. Intérieur : "l'autorité administrative a gravement porté atteinte à la liberté de l'intéressé sans que son action puisse se rattacher à l'application d'un texte législatif ou réglementaire, ou à l'exercice d'un pouvoir lui appartenant" ; 15 février 1995, Osas et Ojo : "le maintien à bord dans ces conditions constitue dès lors une voie de fait à laquelle il doit être mis fin".

14. Le ministère de l'Intérieur déclare qu'il ne cessera de maintenir à bord les «passagers clandestins » que le jour où il aura été condamné pour voie de fait par la Cour de cassation.. Mais comme il ne fait jamais appel des jugements des tribunaux de grande instance qui retiennent la voie de fait, il ne met pas la Cour de cassation en mesure de se prononcer ; il peut donc continuer longtemps.

15. Voir la liste de ces documents et justificatifs, supra note 3.

16. Ord. du 2 nov. 1945, art. 5. Sur les motifs de l'expulsion, voir note 9. L'interdiction du territoire est prononcée, quant à elle, soit par le juge pénal comme peine accessoire à une condamnation pour entrée ou séjour irrégulier en France (art. 19), pour aide à entrée ou séjour irréguliers (art. 21), pour soustraction à une décision de refus d'entrée, à un arrêté d'expulsion ou à une mesure de reconduite à la frontière (art. 27) ou à une décision de remise aux autorités d'un Etat de la Communauté européenne (art. 33), soit par le préfet comme mesure accessoire à un arrêté de reconduite à la frontière (art. 22). 17. Décr. $n^{\circ} 82-442$ du 27 mai 1982, art. 10 et 11.

18. Ord. 2 nov. 1945 , art. 5.

19. Loi no $79-587$ du 11 juill. 1979 relative à la motivation des actes administratifs, art. 3 . 20. En pratique, il est remis à l'étranger non-admis un formulaire, valant notification de la décision, sur lequel est inscrit : «Je renonce au délai d'un jour franc - oui - non (rayer la mention inutile) ».

21. Ord. 2 nov. 1945, art. 5.

22. Voir la liste de ces documents et justificatifs, supra note 3 . 
23. CE 27 sept. 1985, Assoc. France Terre d'asile, Rec. CE, p. 263 ; D. 1986, Somm. p. 278, obs. Ph. Waquet et $\mathrm{F}$. Julien-Laferrière.

24. C. const. 25 févr. 1992, décision n 92-307 DC. Cette même expression est reprise dans la loi du 6 juill. 1992, insérant l'art. 35 quater dans l'ord. du 2 nov. 1945.

25. Décr. du 27 mai 1982, art. 12, déclaré légal par : CE, 27 sept. 1985, Assoc. France Terre d'asile, cité note 24 .

26. Circ. $\mathrm{n}^{\circ}$ NOR/INT/D/92/CC179/C, non publiée.

27. Notamment sans que soit respecté le délai d'un jour franc prévu à l'art. 5 de l'ord. du 2 nov. 1945.

28. Circ. du 9 juill. 1992, précitée.

29. Rapports du ministère de l'Intérieur à la CNCDH pour 1993 et pour le premier semestre de 1994. A la date de la rédaction de cette étude, on ne dispose pas de chiffres plus récents.

30. Sur les conditions de prolongation du maintien et de l'intervention du juge judiciaire, voir infra.

31. En vertu de l'art. R. 46 du code des tribunaux administratifs et des Cours administratives d'appel, le tribunal administratif (TA) territorialement compétent est celui dans le res-sort duquel l'autorité qui a pris la décision de refus d'entrée a son siège. C'est donc le TA dans le ressort duquel se trouve la zone d'attente, sauf si l'étranger non-admis est un demandeur d'asile, hypothèse dans laquelle le TA de Paris est compé-tent, car l'entrée en France ne peut être refusée à un demandeur d'asile que par le ministre de l'Intérieur (v. supra note 21), qui a son siège à Paris.

32. Cass. 2e civ., 9 févr. 1994, Bull. civ. II, nº 53 ; D. 1994, Somm. 254, obs. F. JulienLaferrière.

33. Cette conception du rôle du président du TGI n'est pas partagée par tout le monde. Bien qu'elle fût celle qu'exposa, lors de la discussion de la loi du 6 juillet 1992, Paul Quilès, alors ministre de l'Intérieur, ses successeurs, Charles Pasqua et Jean-Louis Debré, soutinrent, au contraire, que le juge judiciaire ne peut en aucun cas apprécier la demande d'asile. Quant aux juges eux-mêmes, ils divergent également sur ce point. Au sein même des TGI de Bobigny et de Créteil - les deux principalement concernés puisque respectivement compétents pour les aéroports de Roissy et d'Orly†- et de la Cour d'appel de Paris, cer-tains magistrats adoptent la conception large, tandis que d'autres se contentent du « profil bas ». La Cour de cassation n'a, jusqu'à présent, pas tranché la question.

34. Décr. $n^{\circ}$ 92-1333 du 15 déc. 1992, art. 8.

35. Ord. 2 nov. 1945, art. 35 quater.

36. Décr. n 92-1333 du 15 déc. 1992, art. 12.

37. A notre connaissance et selon le service médical de Roissy lui-même, il n'est gardé aucun double des certificats médicaux établis dans ces conditions.

38. Ord. du 2 nov. 1945, art. 35 bis. Voir infra.

39. C. const. 25 févr. 1992, décision $n^{\circ}$ 92-307 DC.

40. Il a été constaté, à l'hôtel Arcade de Roissy, que les serrures avaient été ôtées aux portes des chambres et que les membres d'une même famille étaient parfois séparés. Le ministère de l'Intérieur, alerté par des associations de défense des droits des étrangers, semble avoir donné des instructions pour que ces pratiques cessent.

41. L'OMI - anciennement ONI (Office national de l'immigration) - est un établissement public administratif, créé par l'ord. du 2 nov. 1945 et placé sous la tutelle du ministre chargé des affaires sociales (Direction de la population et des migrations), 
qui a pour mission principale d'assurer l'introduction en France des travailleurs étrangers et des membres de leur famille et de gérer la procédure d'aide à la réinsertion des étrangers rentrant dans leur pays d'origine. Il est également chargé d'effectuer, à la demande du maire, la visite du logement du signataire d'un certificat d'hébergement (ord. du 2 nov. 1945, art. 5-3).

42. Décr. nº 95-507 du 2 mai 1995, J.O. du 4 mai.

43. Publié au J.O. du 15 déc.

44. Séance du 17 juin 1992, JO Sénat, p. 1772.

45. C'est sur la base de ces diverses critiques que l'ANAFÉ a formé contre ce décret un recours en annulation devant le Conseil d'Etat. Ce recours est encore pendant à la date de la rédaction de cette étude.

46. Voir les développements relatifs aux « non admis », supra.

INDEX

Mots-clés : centre de rétention, étrangers, frontières, mobilité

\section{AUTEUR}

\section{FRANÇOIS JULIEN-LAFERRIÈRE}

Professeur à la Faculté Jean Monnet, Directeur du Centre de recherches internationales sur les droits de l'homme (CRIDHOM), Université de Paris Sud. 